\title{
Schlüssellochrettung aus dem PKW (S.IE.G.E.R-Konzept) - Schritt für Schritt
}

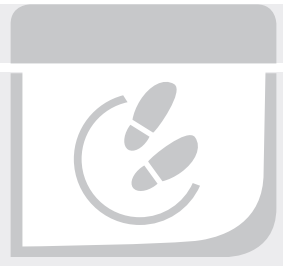

Jens Tiesmeier, Rolf Südmersen, Bernd Bachmann-Mennenga, Thomas Jakob

Im Straßenverkehr kommt es jährlich zu etwa 390000 Unfallverletzten in Deutschland. Allein 2015 starben 3633 Menschen an den Folgen eines Verkehrsunfalls [1]. Bei (potenziell schwerverletzten) Patienten, die zur weiteren medizinischen Versorgung mit professioneller Hilfe aus ihrem PKW befreit werden, gibt es einige Besonderheiten zu beachten.

\section{Einführung}

Häufig sind Verletzte nach Verkehrsunfällen nicht frei zugänglich - etwa, weil sie im Fahrzeug eingeklemmt sind oder Türen sich nicht öffnen lassen. Diese Situationen erfordern ein Gesamtkonzept, das medizinische, technische und taktische Aspekte berücksichtigt. Dazu bedarf es an der Nahtstelle von Rettungsdienst, Feuerwehr und ggf. Polizei der kollegialen Absprache. Gemeinsame Ziele sind der Zugang zum und die Befreiung des Patienten, die Erstbehandlung und der Transport in die Klinik unter Vermeidung weitere Schädigungen ( $\triangleright$ Abb. 1).

\section{Das S.IE.G.E.R.-Konzept}

Da jeder PKW-Unfall und seine Begleitumstände (Patientenzustand, Lage des PKW, Umgebungsbedingungen etc.) einmalig sind, müssen Sie verschiedene Methoden der technischen Rettung beherrschen. Eine Option ist das S.IE.G.E.R-Konzept (S -spricht der Patient?, IE - innere Erkundung, G - genaue Absprache mit der Feuerwehr, E - Entklemmen, R - Rettungsöffnung) für eine schnelle, achsengerechte Rettung. Die einzelnen Schritte können je nach Situation - auch parallel oder in modifizierter Reihenfolge durchgeführt werden.

\section{Schritt 1: Scene Survey - Beurteilung der Situation bei Eintreffen}

Beim Scene Survey verschaffen Sie sich einen Überblick über die Gesamtlage. Im Fokus des Rettungsdienstes stehen die (angenommene) Unfallkinematik, das Schadensmuster und die Zahl der Verletzten.

Ergänzend beurteilt die technische Rettung der Feuerwehr Gefahrenquellen (z. B. Verkehrsabsicherung, Brandgefahr durch auslaufende Betriebsstoffe, Sicherung des Fahrzeugs gegen Wegrollen oder Absturz, muss ein bestehender Brand gelöscht werden?). Dazu gehört das vollständige Anlegen der persönlichen Schutzausrüstung inklusive Helm, um gegen Splitter, Kanten oder Schlageinwirkungen geschützt zu sein.

\section{Schritt 2: S - spricht der Patient? und "Traumahand"}

Um Kontakt aufzunehmen und eine erste Beurteilung des Zustands des Patienten durchzuführen, nähern Sie sich dem Patienten mit ausgestreckter Hand von vorn und sprechen ihn dabei an. Der Patient soll Ihre Hand fixieren, um unbeabsichtigte Drehungen der Halswirbelsäule zu vermeiden.

Die zentrale Frage lautet: „Spricht der Patient - ja oder nein?" Wenn ja, sind Atmung und Kreislauf (noch) möglich und vorhanden. Sie können nun gezielt nach Schmerzen oder Einklemmung fragen und den Patienten betreuen, indem Sie ihm die nachfolgenden Schritte erklären (z. B. „Die Feuerwehr arbeitet am Fahrzeug“, „Das Fahrzeug wird geöffnet“, „Die Kollegen kommen gleich zu Ihnen ins Fahrzeug“).

Wenn nein, schließt sich die Entscheidung an, ob Sie auch ohne Sicherung des PKW direkt versuchen, zum Patienten zu gelangen. Ist die Tür neben dem Patienten blockiert, lässt sich stattdessen vielleicht eine der anderen PKW-Türen öffnen. Alternativ können Fenster oder Heckklappe zerstört werden ( $\triangleright$ Abb. 2).

\section{Schritt 3: Sicherung und Unterbauung des PKW}

Für die Patientenversorgung im PKW muss die Einsatzstelle für Sie sicher sein. Erst nach der Bestätigung der Feuerwehr „Fahrzeug gesichert“ kann der Rettungsdienst in das Fahrzeug einsteigen. 
Durch eine ergänzende Unterbauung des PKW werden die Federwege überbrückt. Das Fahrzeug liegt auf und kann nicht mehr schaukeln; ungewollte Patientenbewegungen werden vermieden. Die Unterbauung erfolgt unter den A-Säulen und vor der Hinterachse. Danach wird die Luft mit einem Ventilausheber aus den Reifen gelassen, und der PKW liegt sicher auf dem Unterbau auf ( $\triangleright$ Abb. 3).

\section{Schritt 4: IE - Innere Erkundung (Patientenzustand nach (c)-ABCDE und Fahrzeugtechnik)}

Mit möglichst großem Abstand zu den Airbags werden gemäß (c)-ABCDE-Schema unmittelbar lebensgefährdende Zustände therapiert und der Zustand des Patienten wird als „kritisch“ oder „unkritisch“ eingestuft. Ergibt sich aus Unfallmechanismus und -folgen der Verdacht auf ein Polytrauma mit notwendiger Schockraumalarmierung, wird eine vollständige Immobilisierung des Patienten vorbereitet ( $\triangleright$ Tab. 1 ).

Bereits initial fixiert ein zweiter innerer Retter den Kopf manuell, während die HWS-Orthese von vorne angelegt wird ( Abb. 4).

Die Deformierung des Fahrzeuginnenraums gibt vor allem bei einem Frontalaufprall Hinweise auf die Verletzungsschwere. Ist das Lenkrad gebrochen, ist der Thorax möglicherweise darauf geprallt (und vielleicht eingeklemmt), im Fußraum könnten Extremitäten eingeklemmt sein. Hat ein Airbag ausgelöst und gibt es Anprallmarken des Gurtsystems?

Solange die Warnblinkanlage funktioniert, ist die Fahrzeugelektrik eine Gefahrenquelle (Airbags, Anlasser, Kurzschluss- und Feuergefahr). Nach dem Abklemmen der Batterie stehen aber elektrische Fensterheber, Sitze oder Lenkräder auch nicht mehr zur Verfügung.

\section{Schritt 5: G - genaue Absprache mit der Feuerwehr}

Richten Sie alle Maßnahmen des Rettungsdienstes und der Feuerwehr am Zustand des Patienten aus. Als nächstes muss der Rettungsweg geplant werden. Teilen Sie allen Beteiligten mit, ob die Rettung sofort, schnell oder schonend erfolgen soll ( $\downarrow$ Tab. 2), ob der Patient eingeklemmt ist, welche Probleme bestehen und welcher Rettungsweg gewählt werden sollte ( $\vee$ Tab. 3).

Die Feuerwehr muss den Rettungsweg planen, ggf. die Entklemmung durchführen oder einen Notausstieg öffnen, falls sich der Zustand des Patienten dramatisch

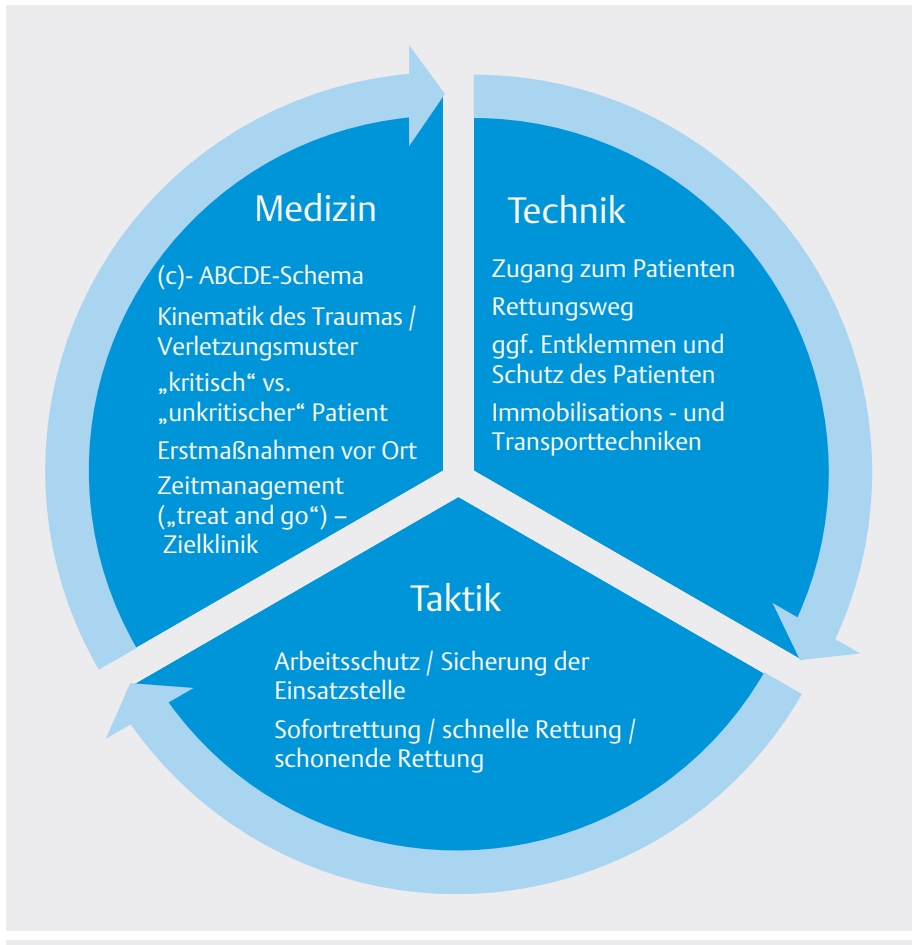

- Abb. 1 Aspekte der patientengerechten Versorgung nach PKW-Unfällen.

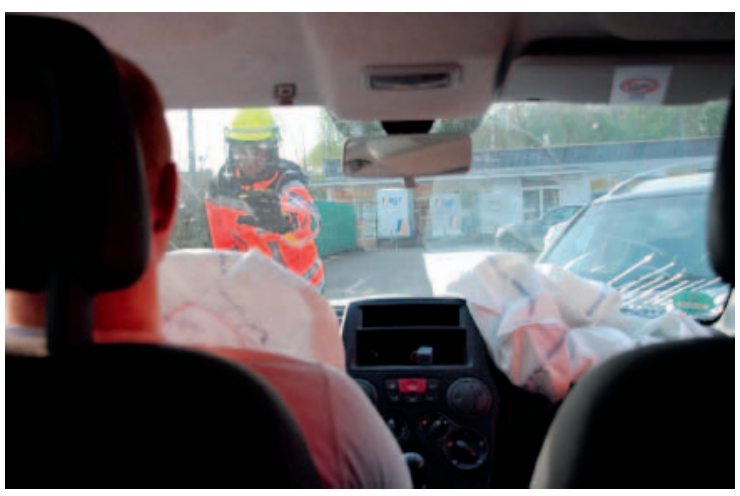

Abb. 2 S - spricht der Patient? und „Traumahand“.

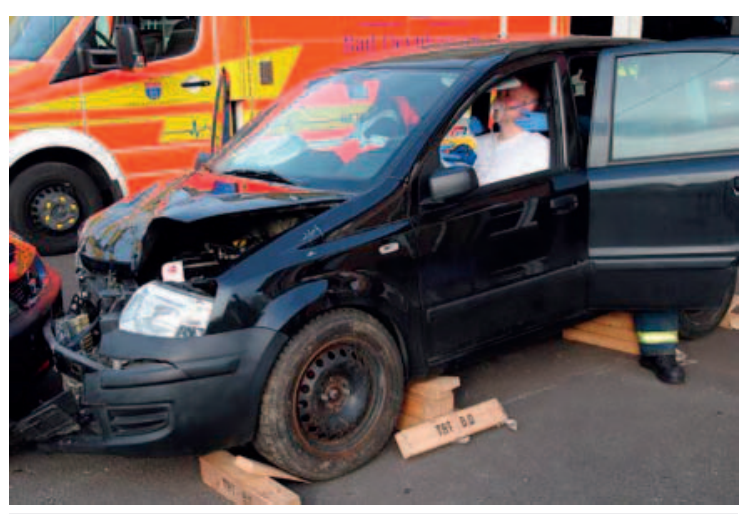

Abb. 3 PKW-Sicherung/-Unterbauung 
- Tab. 1 Arbeitsdiagnose Polytrauma/Schockraumalarmierung [2].

Bei diesen Verletzungen soll das Trauma-Schockraumteam aktiviert werden

- systolischer Blutdruck unter 90 mm Hg (altersadaptiert bei Kindern) nach Trauma

- penetrierende Verletzungen der Rumpf-Hals-Region

- Schussverletzungen der Rumpf-Hals-Region

- GCS unter 9 nach Trauma

- Atemstörungen/Intubationspflicht nach Trauma

- Frakturen von mehr als 2 proximalen Knochen

- instabiler Thorax

- Beckenfrakturen

- Amputationsverletzung proximal der Hände/Füße

- Querschnittsverletzung

- offene Schädelverletzungen

- Verbrennungen $>20 \%$ und Grad $\geq 2 b$
Bei diesen zusätzlichen Kriterien sollte das Trauma-/Schockraumteam aktiviert werden

- Sturz aus über 3 Metern Höhe

- Frontalaufprall mit Intrusion von mehr als $50-75 \mathrm{~cm}$

- Geschwindigkeitsveränderung $\Delta>30 \mathrm{~km} / \mathrm{h}$

- Fußgänger-/Zweiradkollision

- Tod eines Insassen

- Ejektion eines Insassen aus dem Fahrzeug

- Tab.2 Absprachen zum Zeitmanagement einer patientengerechten Rettung [3].

\begin{tabular}{|l|l|l|}
\hline Sofortrettung & Schnelle Rettung & Schonende Rettung \\
\hline $\begin{array}{l}\text { Schnellstmögliche Rettung, weitere } \\
\text { Schädigungen werden toleriert }\end{array}$ & $\begin{array}{l}\text { Zeitfenster } 20-30 \text { Minuten, Beachtung } \\
\text { medizinischer und einsatztaktischer } \\
\text { Aspekte }\end{array}$ & $\begin{array}{l}\text { Die Dauer der Rettung hat eine unter- } \\
\text { geordnete Priorität }\end{array}$ \\
\hline $\begin{array}{l}\text { Beispiele: Reanimation, massives } \\
\text { A-Problem, brennender PKW }\end{array}$ & $\begin{array}{l}\text { Beispiel: Patient ist stabil, achsenge- } \\
\text { rechte Rettung geplant }\end{array}$ & $\begin{array}{l}\text { Beispiel: Isoliertes Wirbelsäulentrauma } \\
\text { ohne Neurologie }\end{array}$ \\
\hline
\end{tabular}

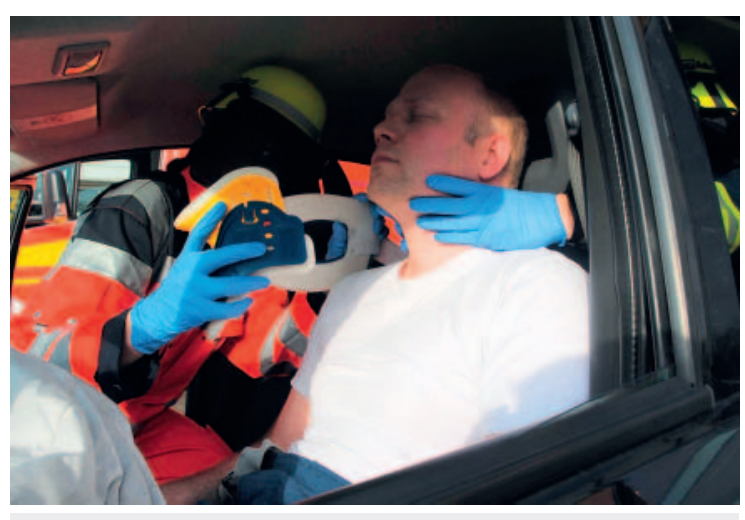

Abb. 4 Innere Erkundung und (c)-ABCDE-Schema.

verschlechtert. Um den Einsatzablauf zu optimieren, müssen alle Beteiligten über das Vorgehen informiert sein.

\section{Schritt 6: R - Rettungsöffnung (Beispiel Schlüssellochrettung)}

Die Schlüssellochrettung als achsengerechte Rettung auf dem Spineboard nutzt die Öffnung des Kofferraums, die eventuell nur minimal durch Spreizen oder Schneiden
\Tab. 3 Mögliche Rettungswege aus dem PKW.

\begin{tabular}{|l|l|l|}
\hline Zur Seite & Nach oben & Nach hinten \\
\hline $\begin{array}{l}\text { Große Seiten- } \\
\text { öfnung, dritte } \\
\text { Tür }\end{array}$ & $\begin{array}{l}\text { Dach abnehmen } \\
\text { oder abklappen, } \\
\text { Dach einsägen } \\
\text { (Fischdose) }\end{array}$ & $\begin{array}{l}\text { Schlüsselloch- } \\
\text { rettung }\end{array}$ \\
\hline
\end{tabular}

erweitert werden muss. Lässt sich die Heckklappe nicht öffnen, kann alternativ die Glasscheibe entfernt werden. Um Ihnen den Zugang zum Patienten zu erleichtern, werden noch intakte Scheiben durch Ankörnung gesplittert. Danach können die Splitter nach außen gedrückt werden. Eine Folie oder Decke dient als weicher Patientenschutz. Die inneren Retter sind dafür (mit-)verantwortlich ( $\triangleright$ Abb. 5).

Beim Schneiden oder Spreizen kommt zusätzlich ein harter Patientenschutz (z. B. Plexiglas) zum Einsatz. Sobald der Patient auch von außen zugänglich ist, senken Sie den Sitz nach hinten ab. Innere und äußere Retter stabilisieren die Wirbelsäule des noch sitzenden Patienten ( Abb. 6). 


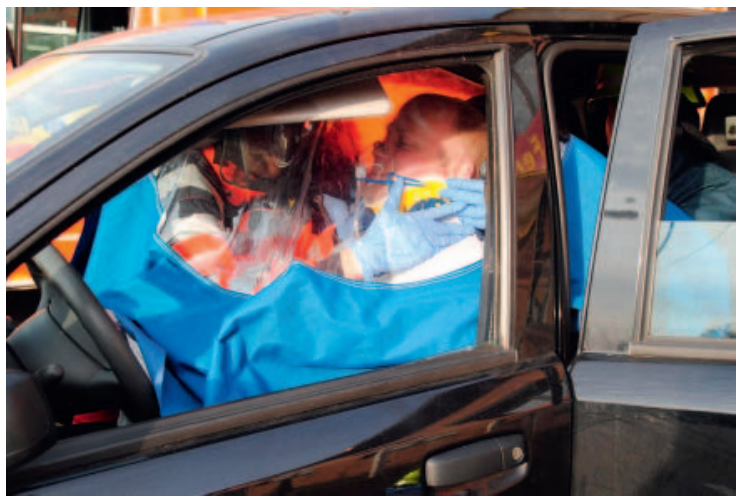

- Abb. 5 Weicher Patientenschutz beim Zersplittern von Glas zur Schaffung eines Zugangs.

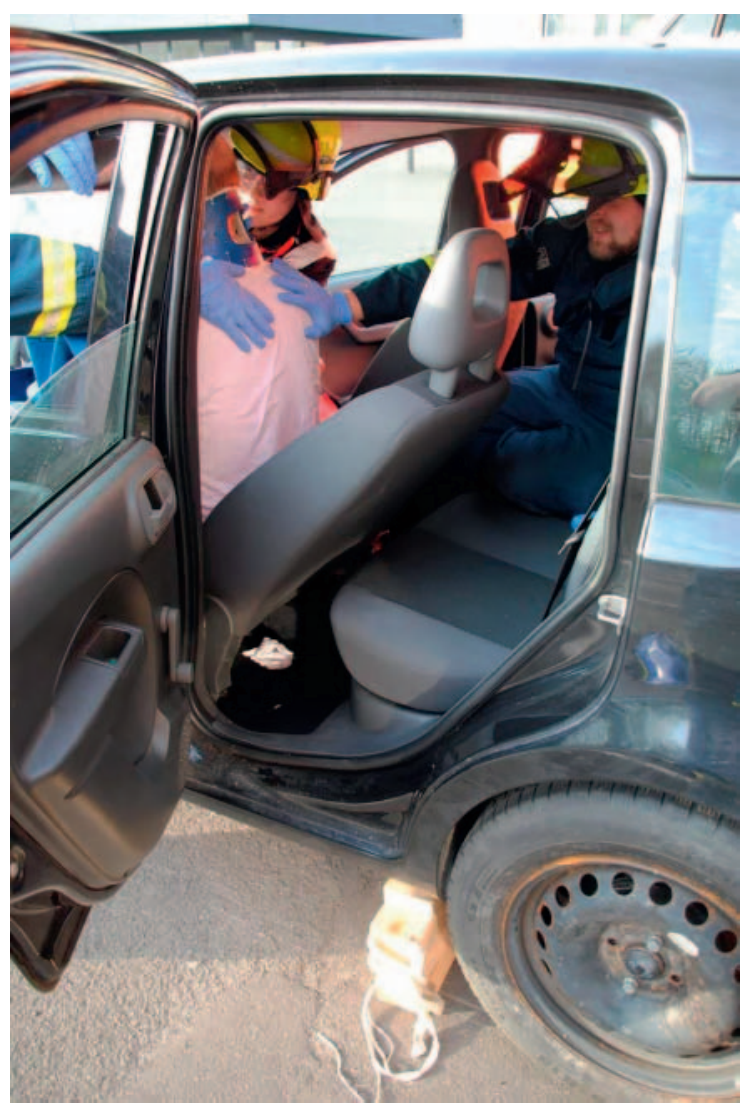

Abb. 6 Sitz absenken und manuelle Stabilisierung der Wirbelsäule.

Zeitgleich schieben Sie das Spineboard über die Hecköffnung in das Wageninnere und legen den Patienten möglichst ohne Bewegung der Wirbelsäule auf dem Rücken darauf ab ( $\triangleright$ Abb. 7, 8).

Achten Sie dabei darauf, dass ein innerer Retter die Halswirbelsäule und den Kopf des Patienten zusätzlich ununterbrochen in Neutralstellung manuell fixiert ( $\triangleright$ Abb. 9).

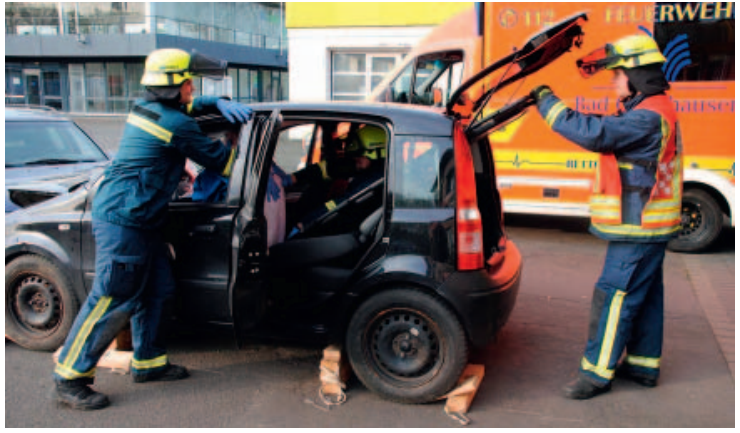

- Abb. 7 Einschieben des Spineboards.

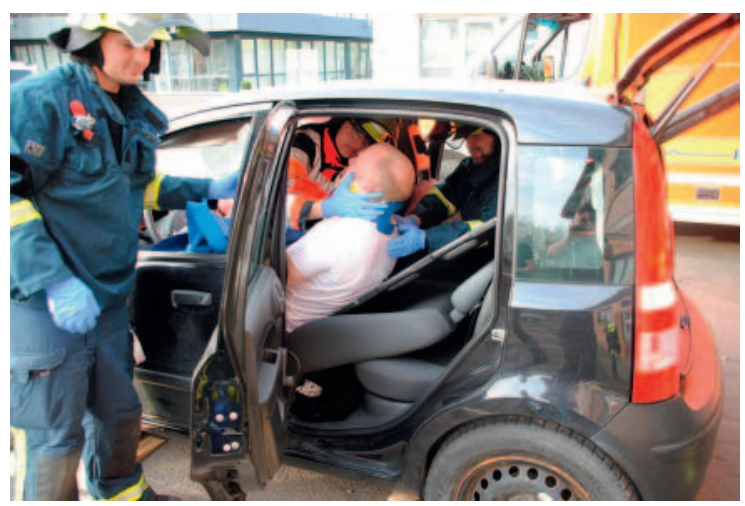

- Abb. 8 Ablegen des Patienten auf dem Spineboard.

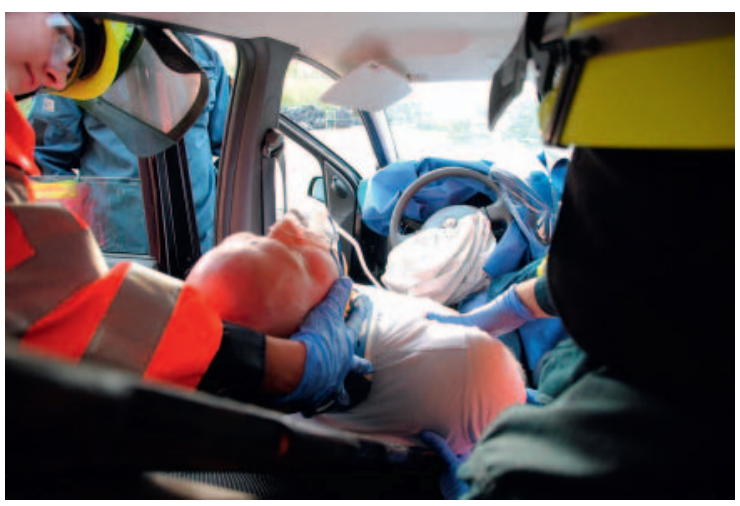

Abb. 9 Bergung des Patienten aus dem Fahrzeug und HWS-Fixierung.

Um den Patienten achsengerecht auf das Spineboard ziehen und aus dem PKW bergen zu können, werden im Optimalfall 6 Helfer benötigt ( $\vee$ Abb. 10).

Wichtig ist eine koordinierte Vorgehensweise: Der Retter an der Kopfposition übernimmt die Führung und gibt die Kommandos. 4 Helfer positionieren sich an Hüfte und Schultern, 1 Helfer fixiert das Spineboard. Ein mögliches Kommando lautet: „Patient $30 \mathrm{~cm}$ auf das Spineboard bei 3: 1-2 - 3.“ Danach wird der Patient gemeinsam 


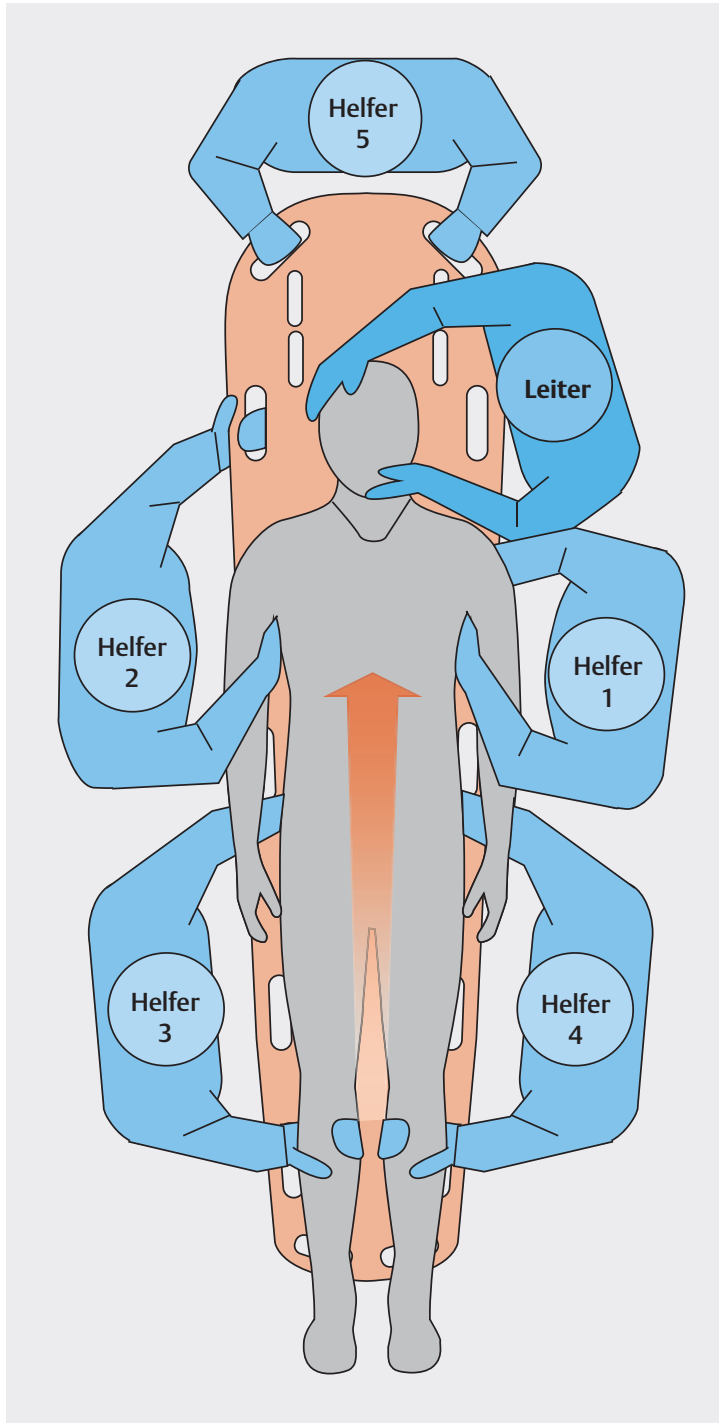

- Abb. 10 Positionierung der Helfer zum Spineboard für die Bergung des Patienten aus dem PKW.

und zeitgleich auf dem Spineboard hochgezogen und gehalten, bis die gewünschte Position erreicht wurde.

Wird der Patient auf dem Spineboard über das Heck aus dem PKW gezogen, muss eine Engstelle passiert werden. Damit der Patient keine Angst bekommt oder in Panik gerät, informieren Sie ihn vor dem Schritt „Tunneln“ und schützen sein Gesicht ( $\triangleright$ Abb. 11).

Zum Abschluss übernimmt ein äußerer Retter die manuelle Fixierung der Halswirbelsäule, bis Headblocks und das Gurtfixiersystem für Spineboards für den weiteren Transport angelegt wurden ( $\triangleright$ Abb. 12).

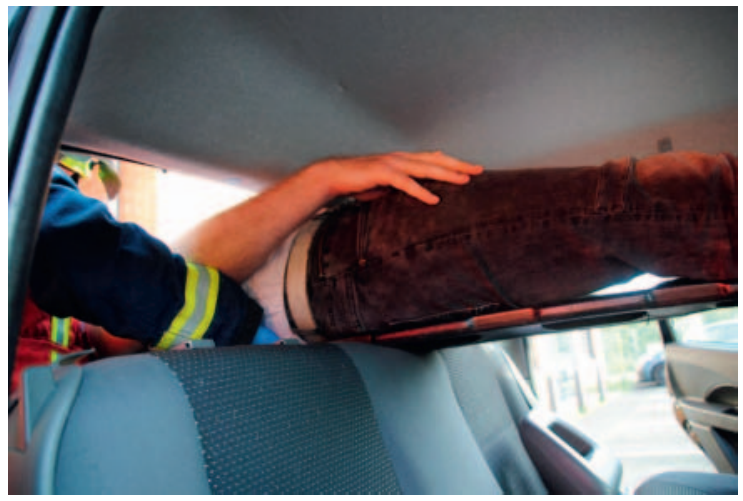

- Abb. 11 „Tunneln“ des Patienten.

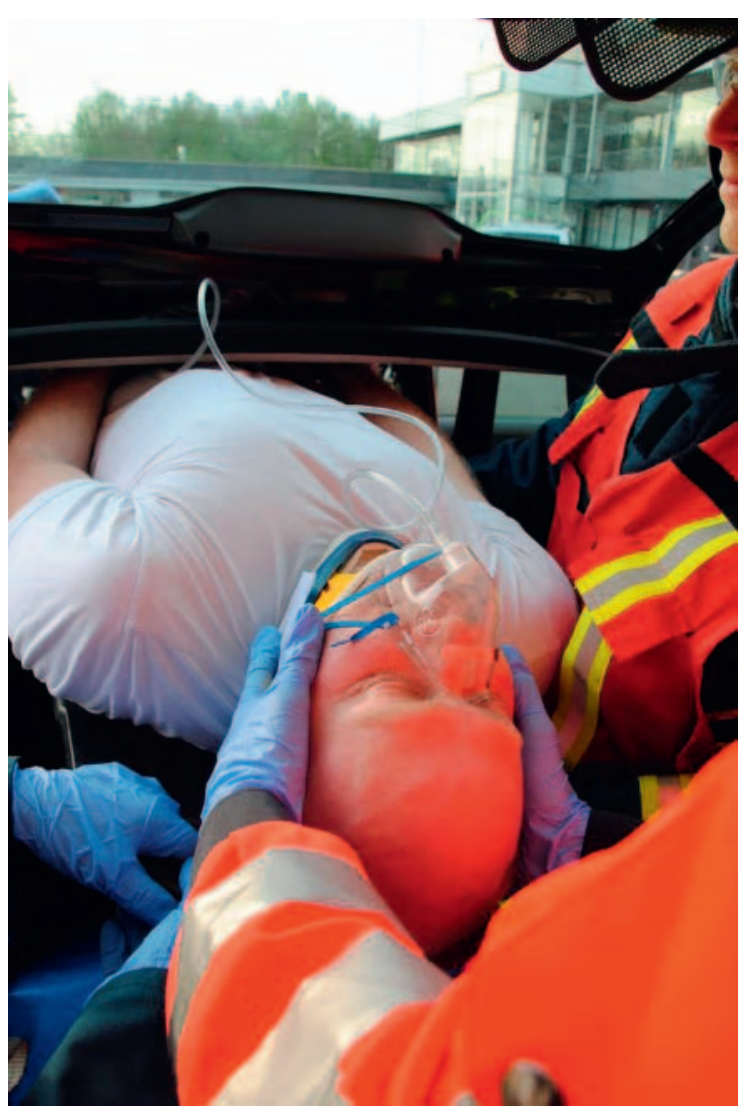

- Abb. 12 Manuelle Fixierung der Halswirbelsäue beim Bergen des Patienten durch die Heckklappe.

\section{Interessenkonflikt}

Die Autoren geben an, dass kein Interessenkonflikt besteht. 


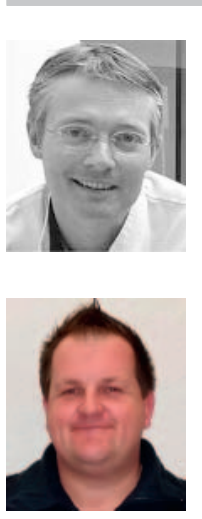

\section{Jens Tiesmeier}

Dr. med. Ärztlicher Leiter der Notarztstandorte Lübbecke-Rahden. Oberarzt am Institut für Anästhesiologie, Intensiv- und Notfallmedizin am Krankenhaus Lübbecke-Rahden der MKKMühlenkreiskliniken.

\section{Rolf Südmersen}

Hauptbrandmeister und Rettungsassistent (Sachgebiet Ausbildung) an der Feuer- und Lehrrettungswache der Stadt Bad Oeynhausen.

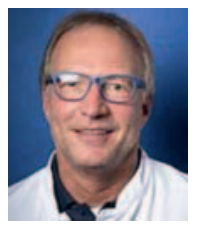

\section{Bernd Bachmann-Mennenga}

Prof. Dr. med. Direktor des Universitätsinstituts für Anästhesiologie, Intensiv- und Notfallmedizin am Johannes Wesling Klinikum Minden, MKK-Mühlenkreiskliniken. Fachvertreter des Querschnittsfachs Notfallmedizin der RuhrUniversität Bochum am Medizin Campus OWL.

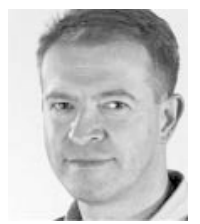

\section{Thomas Jakob}

Dr. med. Ärztlicher Leiter Rettungsdienst im Kreis Herford. Oberarzt an der Universitätsklinik für Anästhesiologie, Operative Intensivmedizin, Rettungsmedizin, Schmerztherapie der Ruhr-Universität Bochum am Klinikum Herford.

\section{Korrespondenzadresse}

Dr. med. Jens Tiesmeier

Institut für Anästhesiologie, Intensiv- und Notfallmedizin

Krankenhaus Lübbecke-Rahden

Virchowstraße 65

32312 Lübbecke

Tel.: ++49/5741/351700

Jens.Tiesmeier@muehlenkreiskliniken.de
Wir danken dem TRT-Bad Oeynhausen und Jan Südmersen (Feuerwehrhandwerk) für die Unterstützung bei der Erstellung der Abbildungen und der fachlichen Beratung.

\section{Literatur}

[1] Bundesanstalt für Arbeitsschutz und Arbeitsmedizin. Unfallstatistik. 2015. Im Internet: https://www.baua.de/DE/Themen/ Arbeitswelt-und-Arbeitsschutz-im-Wandel/Arbeitsweltbericht erstattung/Gesamtunfallgeschehen/Gesamtunfallgesche hen_node.html; Stand: 08.01.2019

[2] Bouillon B, Pieper D. Kurzversion der S3-Leitlinie Polytrauma/ Schwerverletztenbehandlung. AWMF Register-Nr. 012/019. 2017. Im Internet: https://www.awmf.org/uploads/tx_szleitlini en/012-019k_S3_Polytrauma_Schwerverletzten-Behand lung_2017-03.pdf; Stand: 08.01.2019

[3] Technisch-Wissenschaftlicher Beirat (TWB) der Vereinigung zur Förderung des Deutschen Brandschutzes. Merkblatt „Technisch-medizinische Rettung nach Verkehrsunfällen“. 2011. Im Internet: https://www.vfdb.de/fileadmin/download/merk blatt0601.pdf; Stand: 08.01.2019

\section{Bibliografie}

DOI https://doi.org/10.1055/a-0631-4084

retten 2019; 8: 140-145

(c) Georg Thieme Verlag KG, Stuttgart · New York ISSN 2193-2387 